\title{
Black hole superradiance in dynamical spacetime
}

\author{
William E. East,,, Fethi M. Ramazanoğlu, ${ }^{2}$ and Frans Pretorius ${ }^{2}$ \\ ${ }^{1}$ Kavli Institute for Particle Astrophysics and Cosmology, Stanford University, \\ SLAC National Accelerator Laboratory, Menlo Park, California 94025, USA \\ ${ }^{2}$ Department of Physics, Princeton University, Princeton, New Jersey 08544, USA
}

\begin{abstract}
We study the superradiant scattering of gravitational waves by a nearly extremal black hole (dimensionless spin $a=0.99$ ) by numerically solving the full Einstein field equations, thus including backreaction effects. This allows us to study the dynamics of the black hole as it loses energy and angular momentum during the scattering process. To explore the nonlinear phase of the interaction, we consider gravitational wave packets with initial energies up to $10 \%$ of the mass of the black hole. We find that as the incident wave energy increases, the amplification of the scattered waves, as well as the energy extraction efficiency from the black hole, is reduced. During the interaction the apparent horizon geometry undergoes sizable nonaxisymmetric oscillations. The largest amplitude excitations occur when the peak frequency of the incident wave packet is above where superradiance occurs, but close to the dominant quasinormal mode frequency of the black hole.
\end{abstract}

\section{INTRODUCTION}

Despite the fact that spinning black holes (BHs) serve as one-way membranes to causal curves, it is possible to extract rotational energy from them. This can occur through the Penrose process [1] for particles, or by scattering waves off a $\mathrm{BH}$ in an analogous process called superradiance 2-4]. Superradiance occurs for scalar fields, electromagnetic fields, and gravitational waves (GWs), the latter having the strongest possible amplification [5]. This idea has implications across numerous fields from astrophysics - where this energy extraction has been invoked to explain the powering of astrophysical jets in the Blandford-Znajek effect and related processes [6, 7] — to quantum gravity, high-energy physics, and the AdS/CFT correspondence [8] 10].

Superradiance can occur in nongravitational settings as well (e.g. the interaction of electromagnetic radiation with a rotating boundary), and it can be argued that amplification in certain regimes is required by the second law of thermodynamics [11]. For BHs, the analogous argument derives from the connection between horizon area and entropy, and Hawking's area increase theorem [12]. At the linear level, a wave with frequency $\omega$, azimuthal number $m$, and incident energy $\delta E$ will have angular momentum $\delta J=m \delta E / \omega$. The change in a BH's area for a given change in mass and angular momentum is given by $d A=(8 \pi / \kappa)\left(d M-\Omega_{\mathrm{BH}} d J\right)$, where $\Omega_{\mathrm{BH}}$ is the rotational frequency of the BH and $\kappa$ is its surface gravity (we use geometric units with $G=c=1$ throughout). Thus if a wave with frequency $0<\omega<m \Omega_{\mathrm{BH}}$ were wholly absorbed the BH's horizon area would decrease, in violation of the second law. What happens instead is the wave is scattered, gaining energy and angular momentum, and the BH effectively absorbs what globally is counted as negative energy and angular momentum (as also occurs in the Penrose process).

\footnotetext{
* weast@stanford.edu
}

Despite the long history of study of this phenomenon in general relativity, essentially no work has delved beyond the linear level to self-consistently include backreaction, and address how the properties of the spacetime change to balance the energy and angular momentum carried off by the scattered waves. There are several reasons why this is of interest. To begin with, for highly spinning $\mathrm{BHs}$, waves in, and slightly above the superradiant frequency range, carry a ratio of energy to angular momentum such that if they were absorbed they could overspin the $\mathrm{BH}$ [13]. Hence the role of backreaction is important to the question of cosmic censorship. Additionally, superradiance is related to the hypothesized existence of so-called floating orbits [14], originally suggested by Misner, where an object orbiting a spinning $\mathrm{BH}$ extracts energy from the $\mathrm{BH}$ at the same rate it emits energy to infinity via GWs. Recent work suggests that these orbits do not exist at least in the extreme-mass-ratio case 15. (though they may exist for matter coupled to a massive scalar field [16]). However, since very little is known about superradiance in the context of a dynamical spacetime, floating orbits cannot yet be ruled out in general. Finally, superradiance is related to the idea of a black hole "bomb," in which a spinning BH is enclosed in a reflective cavity and perturbed with a superradiant mode, which will grow upon each successive reflection until a sizable fraction of the rotational energy has been transferred to the waves [4, 14] (though cf. [17]). A similar effect occurs with light bosonic fields, where self-interaction due to the mass term plays the role of the confiner [18 21]. If such fields exists, rapidly rotating astrophysical BHs with radii commensurate with the Compton wavelength of the field would be superradiantly unstable, and backreaction would become important to the late-time dynamics.

Of course, since a BH has a limited amount of rotational energy that can be extracted without decreasing the BH's area, it is expected that the efficiency of superradiant amplification will decrease as the amplitude of the incident wave increases. Also, the above thermodynamic arguments used the laws of $\mathrm{BH}$ mechanics for quasistatic processes, hence it is especially interesting to 
explore the behavior of superradiance in the regime where the $\mathrm{BH}$ is highly dynamical. In this paper, we study the superradiant scattering of a gravitational wave packet by a highly spinning $\mathrm{BH}$ using numerical solutions of the full Einstein equations. For low amplitude waves, we find results consistent with linear theory, with an amplification (for the chosen parameters) of $\approx 40 \%$. As the amplitude increases, we find that both the amplification and rotational energy extraction efficiency decrease. During the interaction, the apparent horizon $(\mathrm{AH})$ geometry develops high-amplitude, nonaxisymmetric oscillations, the largest of which occur when the peak frequency of the wave packet is near the least-damped quasinormal (resonant) mode frequency of the $\mathrm{BH}$.

\section{METHODOLOGY}

We solve the field equations in the generalized harmonic formulation, using the code described in 22, 23]. As a gauge choice, we fix the source functions $H^{a}=$ $\square\left(x^{a}\right)$ to be those of the isolated BH. The numerical grid we use has six refinement levels with a grid spacing of $\approx 0.03 M$ on the finest level and $\approx 2.4 M$ in the wave zone, where $M$ is the initial mass of the $\mathrm{BH}$. For select cases, we also run simulations with 0.6 and 0.8 times the resolution to establish convergence.

We construct initial data representing a $\mathrm{BH}$ with an incoming GW packet. We use a BH in Kerr-Schild coordinates with dimensionless spin $a=0.99$. To determine the initial metric perturbation to the $\mathrm{BH}$ spacetime representing the GW, we begin with an ingoing, spin-2 solution to the Teukolsky equation which is characterized by spheroidal harmonic indices $l, m$, and the frequency $\omega$ [5], the angular part of which we numerically calculate as in [24, 25]. For the radial part, we use the approximate asymptotic form [26], which introduces negligible error for a wave packet peaked far from the $\mathrm{BH}$. This gives the value for the Newman-Penrose scalar $\Psi_{0}$, which we then convert into a metric perturbation using the techniques of [26, 27]. We multiply this by a Gaussian envelope in the radial direction peaked at $r_{0} / M=40$ with standard deviation $\sigma / M=10$. To avoid regularity problems at the $\mathrm{BH}$ horizon when transforming the perturbation to Kerr-Schild coordinates, we also multiply by a function that smoothly goes from one to zero at a radius of $\approx 3 M$ from the origin. Finally, we use this solution to specify free data for the constraint equations in the conformal thin-sandwich formulation, which we solve as in [28].

We focus on $l=2$ wave packets and in order to maintain equatorial symmetry, we use the superposition of $(m, \omega)=\left( \pm 2, \pm \omega_{0}\right)$ solutions. This $l$ and $m$, along with our choice of $\omega_{0} M=0.75$, is chosen to give near maximal amplification given our frequency spread. We consider wave packets with a range of amplitudes. The lowest amplitude wave has a mass of $\approx 5.8 \times 10^{-4} M$. Normalizing to this lowest value, the other waves have amplitudes $A=2,4,8$, and 12 times this value. The mass of a wave packet scales as $A^{2}$ and the largest amplitude case has a mass of $0.098 M$. We perform resolution studies for the $A=4$ and $A=12$ cases. We also perform simulations with $\omega_{0} M=0.865$ and $\omega_{0} M=1$ (near and above the maximum superradiant frequency), and amplitudes chosen to give the same mass as the $A=12$ case. We measure the outgoing GW radiation resulting from the interaction of the wave packet with the $\mathrm{BH}$ by calculating the Newman-Penrose scalar $\Psi_{4}$ (see e.g. 29]) and monitor the $\mathrm{BH} \mathrm{AH}$ during the scattering process.

\section{RESULTS}

For the smaller amplitude cases $(A \lesssim 4)$ with $\omega_{0} M=$ 0.75 , the energy and angular momentum of the outgoing GWs are approximately proportional to the ingoing values (i.e., they scale with $A^{2}$ ) as expected in linear perturbation theory. Using the difference between the $\mathrm{BH}$ horizon mass and the ADM mass as a measure of the initial mass of the GW wave packet, we estimate in this regime (in particular for $A=4$ ) the outgoing GW carries an extra $44 \pm 7 \%$ more energy than the ingoing GW. This is consistent with the $\approx 40 \%$ expected from linear perturbation theory [5] for such an energy spectrum. However, as seen in Fig. 1 for larger amplitude cases the scaling begins to break down and there is less energy at higher frequencies relative to the low amplitude cases. This trend is consistent with the fact that (as we discuss below) the spin of the $\mathrm{BH}$ is noticeably decreasing for these cases and $\mathrm{BHs}$ with lower spins are less superradiant at higher frequencies.

The decreasing amplification with increasing wave amplitude is also illustrated in Fig. 2. We show how the total radiated energy $E_{\mathrm{GW}}$ scales with the ingoing amplitude. After dividing out by the contribution which goes as $A^{2}$, we find that the measured energy is well fit by including an additional $A^{4}$ term which decreases this quantity at high amplitudes. We note that the convergence study indicates that the truncation error in the ratio of quantities (e.g. $E_{\mathrm{GW}}$ ) from simulations with different $A$ is noticeably smaller than the absolute error in the quantity for either $A$ (suggesting that the leading-order error scales with the magnitude of the quantity). We also show the ratio of $E_{\mathrm{GW}}$ to the radiated angular momentum $J_{\mathrm{GW}}$. For small amplitudes this ratio is very close to the value given by the dominant frequency of the ingoing $\mathrm{GW}, \omega_{0} / m=0.375 / M$, though for higher amplitudes this decreases, with the trend also well fit by a quadratic expression in $A$. In the bottom panel of Fig. 2 we show the estimated change in the $\mathrm{BH}$ parameters using these extrapolations. For comparison, we also show the change that would occur if the energy and angular momentum absorbed by the $\mathrm{BH}$ followed the linear scaling for all A. Assuming cosmic censorship, this by itself implies the need for nonlinear corrections, as otherwise it predicts $M_{\text {ir }}$ should begin to decrease beyond $A \approx 22$. Moreover, viewed as a quasistatic process, this behavior would be- 

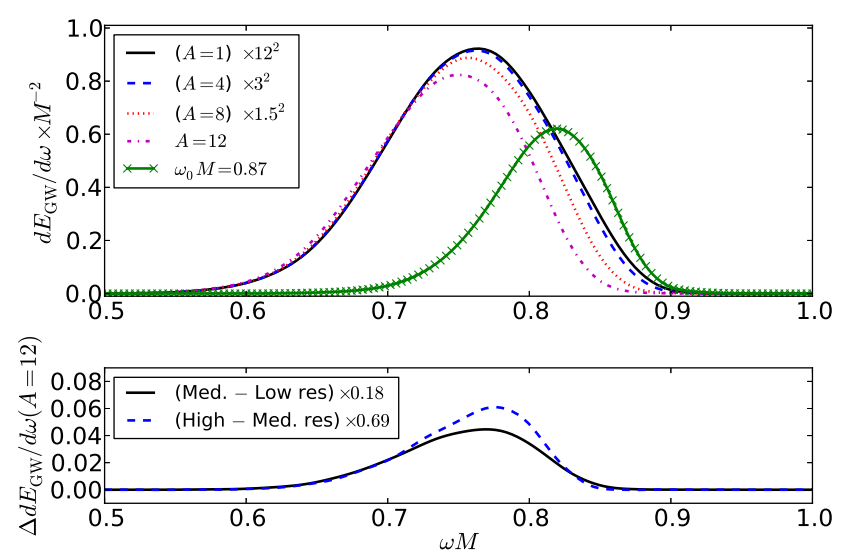

FIG. 1. Top: Energy spectrum of outgoing GWs after the scattering of an ingoing GW packet with central frequency $\omega_{0} M=0.75$ and various amplitudes. The lower amplitude waves have been rescaled according to their leading-order dependence on ingoing amplitude. We also show a case with $\omega_{0} M=0.87$ and the same initial energy as the $A=12$ case. Bottom: Truncation error for the $A=12$ case, consistent with between third- and fourth-order convergence (the error has been scaled assuming the latter).

gin to be problematic at even lower amplitudes $(A \approx 13)$ where $\Delta M_{\text {ir }}$ reaches a maximum and starts to decrease. This occurs when the rotational frequency of the final $\mathrm{BH}$ (after losing energy to the $\mathrm{GW}$ ) is $<\omega_{0} / m$. The fit to the simulation results, on the other hand, extends the range in $A$ where $\Delta M_{\mathrm{ir}}$ shows a monotonic increase. This trend eventually reverses as well, implying (again assuming cosmic censorship) that higher-order nonlinear terms would come into play. Note that for $A \gtrsim 38$ the $\mathrm{GW}$ energy will exceed that of the $\mathrm{BH}$, and the problem becomes one of collapse of a $\mathrm{GW}$ perturbed by a $\mathrm{BH}$.

In superradiant scattering, the $\mathrm{BH}$ loses some of its rotational energy $E_{\text {rot }}=M_{\mathrm{BH}}-M_{\mathrm{ir}}$, part of which is carried away by the wave, and part of which goes into increasing $M_{\mathrm{ir}}$, and hence is no longer extractable. This process can be viewed as having an efficiency $\eta=$ $\Delta M_{\mathrm{BH}} / \Delta E_{\text {rot }}$. In Fig. 3 we show the efficiency obtained using the fits in Fig. 2 The efficiency does initially increase with total extracted energy, though it eventually reaches a maximum. It is also always below the efficiency that would occur if the $\mathrm{BH}$ underwent a quasistatic process with $d J=\left(m / \omega_{0}\right) d M$ (also shown), reminiscent of the fact that in thermodynamics, maximum efficiency is achieved only in a quasistatic process (e.g. a Carnot cycle).

In the extracted GWs we also see an indication of nonlinear mode coupling, predominantly in the $l=|m|=4$ modes (here we refer to spin weight -2 spherical, not spheroidal, components), which are absent in the ingoing GW (as verified by examining $\Psi_{0}$ ). These modes oscillate at twice the frequency of the $l=|m|=2$ modes. The numerical errors on the higher modes are large (and indi-
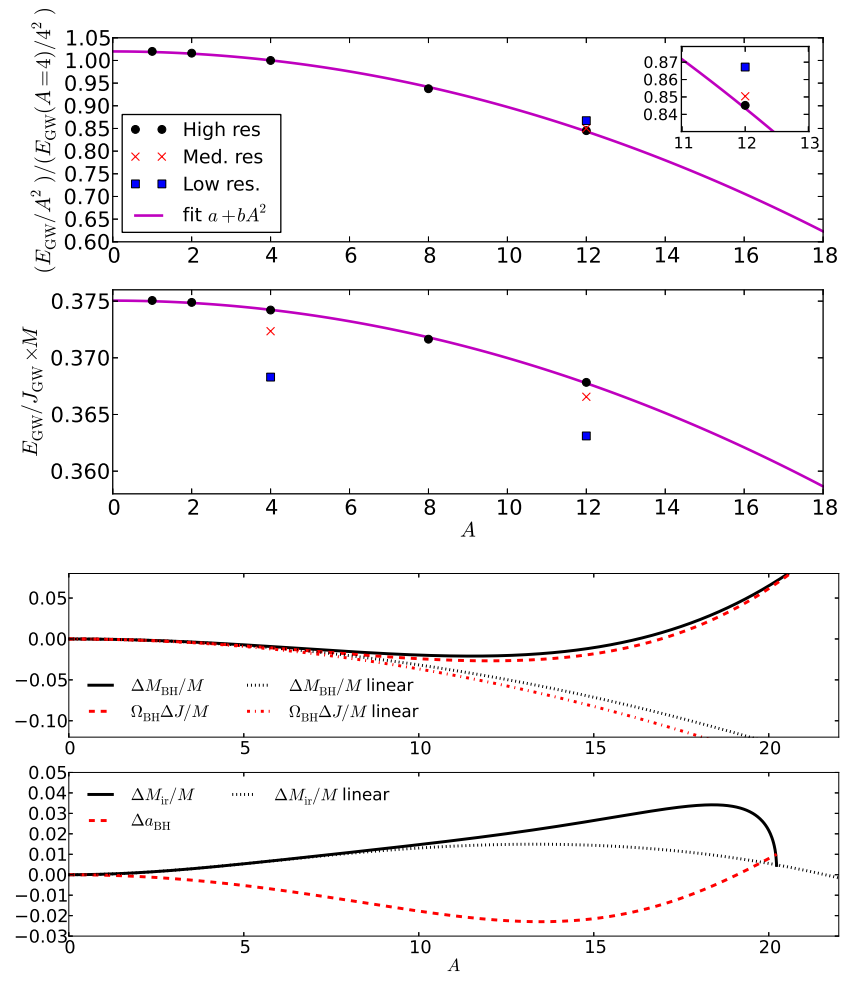

FIG. 2. Top: The energy in outgoing GWs for different values of the ingoing amplitude $A$, relative to the value obtained by scaling the $E_{\mathrm{GW}}$ value at $A=4$ as $A^{2}$, as predicted by linear theory. Below that is shown the ratio of the energy to angular momentum of the outgoing GWs. The curves are fits of the form $a+b A^{2}$. We indicate the numerical error by showing the results from the low and medium resolution runs at $A=4$ and 12. Bottom: The changes in the $\mathrm{BH}$ quantities of total mass, angular momentum times the initial BH rotational frequency (upper panel), as well as irreducible mass, and dimensionless spin (lower panel) implied by the above fits. We also show the changes in the $\mathrm{BH}$ that would occur if the outgoing GWs were always proportional to the ingoing GWs (labeled linear, i.e., ignoring the $A^{2}$ term of the fit shown in the top two panels).

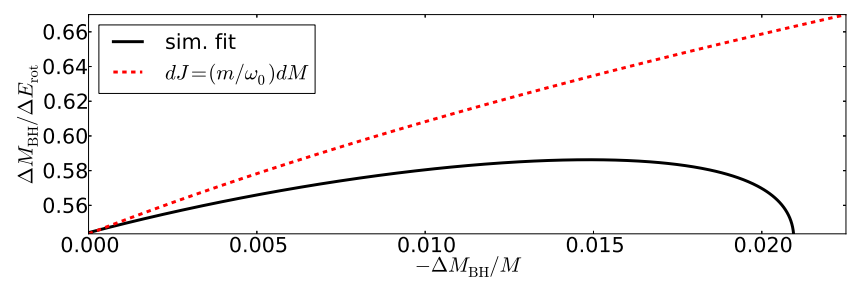

FIG. 3. The efficiency of BH energy extraction $\eta$, as a function of the energy extracted from the $\mathrm{BH}$, that is implied by the fit to simulation results in Fig. 2 (lower amplitude branch). We also show the efficiency expected for a process where the change in $\mathrm{BH}$ angular momentum to $\mathrm{BH}$ mass is given by $d J=\left(m / \omega_{0}\right) d M$. 
cate an underestimate), but the simulations indicate that the ratio of the energy in the $(l, m)=(4,4)$ component to the $(2,2)$ component scales as $A^{2}$, with $E_{44} / E_{22} \sim 0.03$ at $A=12$.

In Fig. 4 we show the behavior of the $\mathrm{BH} \mathrm{AH}$ for the largest amplitude $(A=12)$ case. For comparison, we also show two other cases with the same mass wave packet but with higher frequencies. In the $\omega_{0} M=0.87$ case about $1 / 3$ of the ingoing GW energy is absorbed, with the absorbed energy coming from the component of the wave packet with frequency above $m \Omega_{\mathrm{BH}}$ (see Fig. 11). For $\omega_{0} M=1$, the $\mathrm{GW}$ is almost entirely absorbed by the $\mathrm{BH}$. In contrast, the superradiance of the $\omega_{0} M=0.75$ case is borne out by the overall decrease in angular momentum $J_{\mathrm{BH}}$ and mass $M_{\mathrm{BH}}$ of the $\mathrm{BH}$ shown in the top panel. We note that during the highly dynamic phase of the evolution, when the peak of the GW is incident on the $\mathrm{BH}$ there is no (even approximate) axisymmetric Killing vector, and hence no way of unambiguously defining $J_{\mathrm{BH}}$ (and therefore $M_{\mathrm{BH}}$, which we compute from $J_{\mathrm{BH}}$ and the irreducible mass $M_{\mathrm{ir}}$ ) in a coordinate-independent fashion. We simply calculate $J_{\mathrm{BH}}$ and $M_{\mathrm{BH}}$ using the axisymmetric Killing vector of the isolated $\mathrm{BH}$ spacetime, and so some caution must be taken interpreting these quantities during the interaction.

Despite the opposite sign of the change in $\mathrm{BH}$ mass, the $\omega_{0} M=0.75$ and 0.87 cases show a similar small increase in irreducible mass. (We note that for these cases, when the $\mathrm{BH}$ is highly distorted around $t \approx 50 \mathrm{M}$, the AH finding algorithm is not always able to locate the horizon to the desired tolerance, and the quantities computed around then are less accurate; this accounts for the noisiness of these curves around that time.) In the middle panel of Fig. 4 we show the ratio of the proper polar (as measured arbitrarily in the $y=0$ plane) and equatorial circumferences $C_{\mathrm{p}} / C_{\mathrm{eq}}$ of the $\mathrm{AH}$ indicating that the $\mathrm{AH}$ is becoming distorted from its axisymmetric shape and oscillating. The horizon distortions are further illustrated by the fact that in all three cases the maximum Ricci scalar curvature of the AH 2-surface $R_{\mathrm{AH}}$ temporarily increases above the value of an unperturbed, $a=1 \mathrm{BH}$. The maximum value of $R_{\mathrm{AH}}$ occurs on the equator (and the minimum at the poles), as it does for an isolated spinning $\mathrm{BH}$, though these AHs show large deviations from axisymmetry.

As can be seen in Fig. 4, the largest distortions of the $\mathrm{AH}$, in terms of oscillations of $C_{\mathrm{p}} / C_{\mathrm{eq}}$ and $R_{\mathrm{AH}}$, occur not for the case that is overall superradiant, nor for the case where the BH's mass and angular momentum change the most, but for $\omega_{0} M=0.87$. This is presumably related to the fact that, for this case, the energy is peaked near the resonant frequency of the $\mathrm{BH}$, which approaches $m \Omega_{\mathrm{BH}}$ from above as $a \rightarrow 1$ [30].
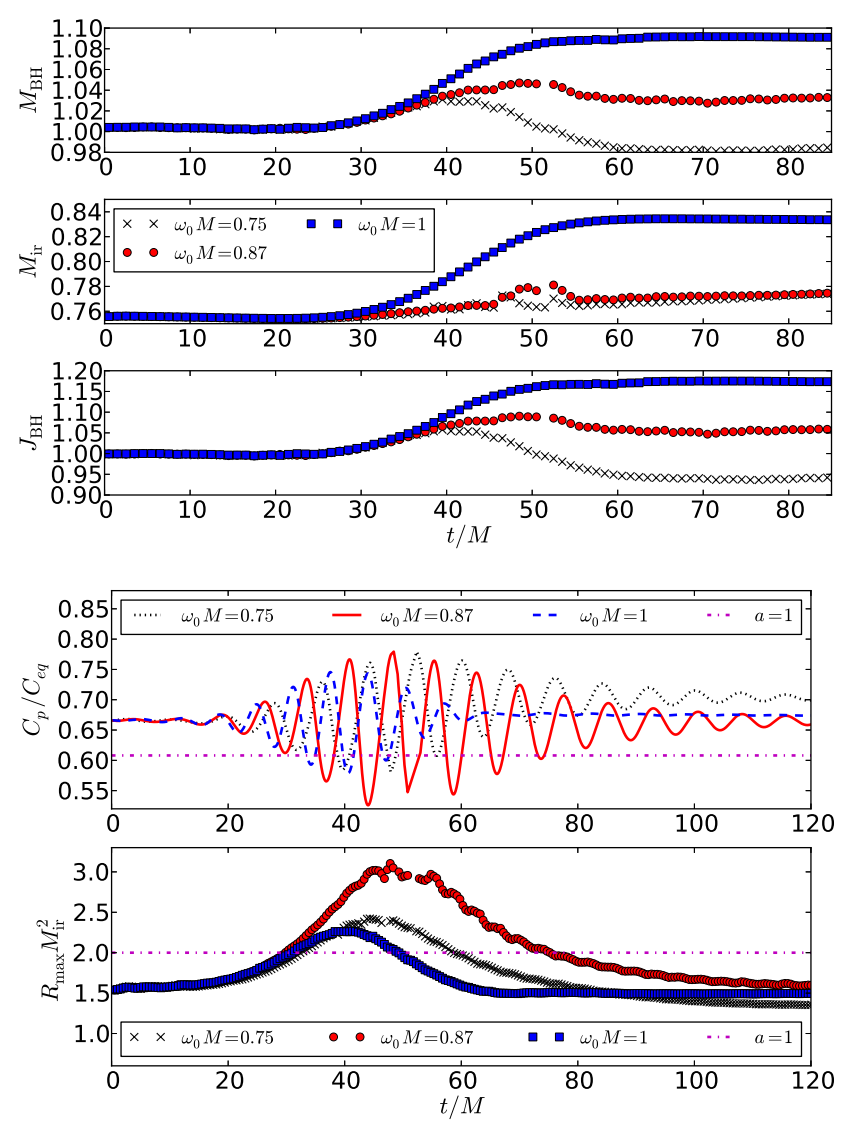

FIG. 4. AH quantities during interaction with different frequency GW packets, each with initial mass $\approx 0.1 M$. Shown (in units where $M=1$ ) are the mass, irreducible mass, and angular momentum of the $\mathrm{BH}$ (top panel), the ratio of proper polar to equatorial circumference (middle panel), and the maximum Ricci curvature of the horizon surface (bottom panel). For the latter two quantities, we also show the values of an unperturbed $\mathrm{BH}$ with extremal spin.

\section{CONCLUSIONS}

In this paper, we presented results from full solutions of the Einstein equations describing the superradiant scattering of GW packets by a highly spinning BH. To our knowledge this is the first study of this phenomenon beyond the linear and quasistatic regimes. We demonstrated the reduction in the BH's rotational energy resulting from this effect. For large amplitude waves we found that backreaction serves to reduce the amplification of the scattered waves as well as reduce the higher frequency content of the wave packet. The net result is a decrease in the efficiency of energy extraction, as expected for the $\mathrm{BH}$ to avoid losing more energy than allowed by Hawking's area theorem. Due to technical difficulties in following what becomes a highly dynamical and distorted $\mathrm{AH}$ (a necessity for stable evolution with our excision method), we were unable to study the 
interaction of waves with initial energy $\gtrsim 0.1 M$. This would be an interesting area for future work, as the extrapolation in Fig. 2 suggests then higher-order effects are needed to prevent the $\mathrm{BH}$ from becoming superextremal. We suspect that with the slicing employed here the intrinsic geometry of the event horizon is well mimicked by that of the AH, though it would be interesting to explicitly study the event horizon structure to confirm this.

We thank Amos Ori and Mihalis Dafermos for stimulating discussions that initiated this work. This research was supported by NSF Grants No. PHY-1065710, No. PHY1305682, and the Simons Foundation (FP). Simulations were run on the orbital cluster at Princeton University.
[1] R. Penrose, Riv. Nuovo Cimento 1, 252 (1969).

[2] C. W. Misner, Phys. Rev. Lett. 28, 994 (1972).

[3] A. A. Starobinskij, Zhurnal Eksperimentalnoi i Teoreticheskoi Fiziki 64, 48 (1973).

[4] Y. B. Zel'Dovich, Soviet Journal of Experimental and Theoretical Physics 35, 1085 (1972).

[5] S. A. Teukolsky and W. H. Press, Astrophys. J. 193, 443 (1974).

[6] R. D. Blandford and R. L. Znajek, Mon. Not. R. Astron. Soc. 179, 433 (1977).

[7] R. Ruffini and J. R. Wilson, Phys.Rev. D12, 2959 (1975).

[8] B. S. DeWitt, Physics Reports 19, 295 (1975).

[9] S. W. Hawking and H. S. Reall, Phys. Rev. D 61, 024014 (1999).

[10] I. Bredberg, T. Hartman, W. Song, and A. Strominger, Journal of High Energy Physics 2010, 1 (2010).

[11] J. D. Bekenstein and M. Schiffer, Phys.Rev. D58, 064014 (1998), gr-qc/9803033.

[12] J. D. Bekenstein, Phys. Rev. D 7, 949 (1973).

[13] K. Düztaş and İ. Semiz, Phys. Rev. D 88, 064043 (2013), 1307.1481.

[14] W. H. Press and S. A. Teukolsky, Nature (London)238, 211 (1972).

[15] S. J. Kapadia, D. Kennefick, and K. Glampedakis, Phys. Rev. D 87, 044050 (2013).

[16] V. Cardoso, S. Chakrabarti, P. Pani, E. Berti, and L. Gualtieri, Physical Review Letters 107, 241101
(2011), 1109.6021.

[17] H. Witek et al., Phys.Rev. D82, 104037 (2010), 1004.4633 .

[18] T. Damour, N. Deruelle, and R. Ruffini, Lettere Al Nuovo Cimento Series 2 15, 257 (1976).

[19] S. L. Detweiler, Phys.Rev. D22, 2323 (1980).

[20] T. Zouros and D. Eardley, Annals Phys. 118, 139 (1979).

[21] H. Witek, V. Cardoso, A. Ishibashi, and U. Sperhake, Phys.Rev. D87, 043513 (2013), 1212.0551.

[22] F. Pretorius, Class. Quantum Grav. 22, 425 (2005), arXiv:gr-qc/0407110.

[23] W. E. East, F. Pretorius, and B. C. Stephens, Phys.Rev. D85, 124010 (2012), 1112.3094.

[24] E. Berti, V. Cardoso, and M. Casals, Phys. Rev. D73, 024013 (2006), gr-qc/0511111.

[25] E. W. Leaver, Royal Society of London Proceedings Series A 402, 285 (1985).

[26] A. Ori, Phys. Rev. D67, 124010 (2003), gr-qc/0207045.

[27] C. O. Lousto and B. F. Whiting, Phys. Rev. D66, 024026 (2002), gr-qc/0203061.

[28] W. E. East, F. M. Ramazanoglu, and F. Pretorius, Phys. Rev. D86, 104053 (2012), 1208.3473.

[29] L. L. Smarr, Gauge conditions, radiation formulae and the two black hole collision, in Sources of Gravitational Radiation, edited by L. L. Smarr, p. 245, Cambridge University Press, Cambridge, U.K., 1979.

[30] S. L. Detweiler, Proc. R. Soc. London Ser. A 352, 381 (1977). 